\title{
Corrigendum: Major Stress-Related Symptoms During the Lockdown: A Study by the Italian Society of Psychophysiology and Cognitive Neuroscience
}

\author{
Sara Invitto ${ }^{* *}$, Daniele Romano ${ }^{2}$, Francesca Garbarini ${ }^{3}$, Valentina Bruno ${ }^{3}$, \\ Cosimo Urgesi ${ }^{4}$, Giuseppe Curcio ${ }^{5}$, Alberto Grasso ${ }^{1}$, Maria Concetta Pellicciari ${ }^{6}$, \\ Giacomo Koch ${ }^{7,8}$, Viviana Betti ${ }^{7,9}$, Mirta Fiorio ${ }^{10}$, Emiliano Ricciardi ${ }^{11}$, \\ Marina de Tommaso $^{12}$ and Massimiliano Valeriani ${ }^{13}$
}

\section{OPEN ACCESS}

Approved by:

Frontiers Editorial Office,

Frontiers Media SA, Switzerland

*Correspondence:

Sara Invitto

sara.invitto@unisalento.it

Specialty section:

This article was submitted to

Public Health Policy,

a section of the journal

Frontiers in Public Health

Received: 18 May 2021 Accepted: 20 May 2021

Published: 11 June 2021

Citation:

Invitto $S$, Romano D, Garbarini $F$,

Bruno V, Urgesi C, Curcio G,

Grasso A, Pellicciari MC, Koch G,

Betti V, Fiorio M, Ricciardi E, de Tommaso $M$ and Valeriani M (2021) Corrigendum: Major Stress-Related Symptoms During the Lockdown: A

Study by the Italian Society of

Psychophysiology and Cognitive Neuroscience.

Front. Public Health 9:711132.

doi: 10.3389/fpubh.2021.711132
${ }^{1}$ INSPIRE LAB - Laboratory of Cognitive and Psychophysiological Olfactory Processes, Department of Biological and Environmental Sciences and Technologies, University of Salento, Lecce, Italy, ${ }^{2}$ Department of Psychology, University of Milano-Bicocca, Milan, Italy, ${ }^{3}$ Manibus Lab, Department of Psychology, University of Torino, Torino, Italy, ${ }^{4}$ Department of Languages and Literatures, Communication, Education, and Society, University of Udine, Udine, Italy, ${ }^{5}$ Dipartimento di Scienze Cliniche Applicate e Biotecnologiche, Università Degli Studi Dell'Aquila, L'Aquila, Italy, ${ }^{6}$ UniCamillus, Saint Camillus International University of Health Science, Rome, Italy, ${ }^{7}$ Fondazione Santa Lucia IRCCS, Rome, Italy, ${ }^{8}$ Dipartimento di Neuroscienze e Riabilitazione, Università di Ferrara, Ferrara, Italy, ${ }^{9}$ Department of Psychology, Sapienza University of Rome, Rome, Italy, ${ }^{10}$ Department of Neurosciences, Biomedicine and Movement Sciences, University of Verona, Verona, Italy, ${ }^{11}$ IMT School for Advanced Studies, Lucca, Italy, ${ }^{12}$ Department of Basic Medical Science, Neuroscience and Sense Organs, University of Bari Aldo Moro, Bari, Italy, ${ }^{13}$ Neurology Unit, Bambino Gesù Hospital, Rome, Italy

Keywords: Covid-19, pain, sleep habits, olfactory perception, lockdown, stress, anxiety symptoms

\section{A Corrigendum on}

Major Stress-Related Symptoms During the Lockdown: A Study by the Italian Society of Psychophysiology and Cognitive Neuroscience

by Invitto, S., Romano, D., Garbarini, F., Bruno, V., Urgesi, C., Curcio, G., et al. (2021). Front. Public Health 9:636089.doi: 10.3389/fpubh.2021.636089

An author name was incorrectly spelled as Giacomo Kock. The correct spelling is Giacomo Koch.

In the published article, there was an error in affiliation 7. Instead of "Dipartimento di Neuroscienze, Policlinico Tor Vergata, Rome, Italy," it should be "Fondazione Santa Lucia IRCCS Rome, Italy and Dipartimento di Neuroscienze e Riabilitazione, Università di Ferrara, Ferrara, Italy".

In the published article, there was an error regarding the affiliation(s) for Giacomo Koch. As well as having affiliation(s) 7, they should also have affiliation $\mathbf{8}$.

The authors apologize for this error and state that this does not change the scientific conclusions of the article in any way. The original article has been updated.

Copyright (c) 2021 Invitto, Romano, Garbarini, Bruno, Urgesi, Curcio, Grasso, Pellicciari, Koch, Betti, Fiorio, Ricciardi, de Tommaso and Valeriani. This is an open-access article distributed under the terms of the Creative Commons Attribution License (CC BY). The use, distribution or reproduction in other forums is permitted, provided the original author(s) and the copyright owner(s) are credited and that the original publication in this journal is cited, in accordance with accepted academic practice. No use, distribution or reproduction is permitted which does not comply with these terms. 